\title{
Optimal Error Estimates for the Fully Discrete Interior Penalty DG Method for the Wave Equation
}

\author{
Marcus J. Grote · Dominik Schötzau
}

Received: 31 May 2008 / Accepted: 29 August 2008 / Published online: 18 September 2008

(C) Springer Science+Business Media, LLC 2008

\begin{abstract}
In Grote et al. (SIAM J. Numer. Anal., 44:2408-2431, 2006) a symmetric interior penalty discontinuous Galerkin (DG) method was presented for the time-dependent wave equation. In particular, optimal a-priori error bounds in the energy norm and the $L^{2}$-norm were derived for the semi-discrete formulation. Here the error analysis is extended to the fully discrete numerical scheme, when a centered second-order finite difference approximation ("leap-frog" scheme) is used for the time discretization. For sufficiently smooth solutions, the maximal error in the $L^{2}$-norm error over a finite time interval converges optimally as $O\left(h^{p+1}+\Delta t^{2}\right)$, where $p$ denotes the polynomial degree, $h$ the mesh size, and $\Delta t$ the time step.
\end{abstract}

Keywords Discontinuous Galerkin methods · Finite element methods · Wave equation · Interior penalty method $\cdot$ Leap-frog scheme

\section{Introduction}

In [20] a symmetric interior penalty discontinuous Galerkin method was presented for the time-dependent wave equation in its standard second-order form. There in particular, optimal a-priori error bounds in the energy norm and the $L^{2}$-norm were derived for the semidiscrete formulation, where the time dependence is kept continuous. Clearly time must also be discretized in practice, which leads to additional errors at every time step. Therefore, in

The research of M.J. Grote was supported in part by the Swiss National Science Foundation (SNF). The research of D. Schötzau was supported in part by the Natural Sciences and Engineering Research Council of Canada (NSERC).

M.J. Grote ( $\varangle)$

Department of Mathematics, University of Basel, Rheinsprung 21, 4051 Basel, Switzerland

e-mail: Marcus.Grote@unibas.ch

D. Schötzau

Mathematics Department, University of British Columbia, 121-1984 Mathematics Road,

Vancouver V6T 1Z2, Canada

e-mail: schoetzau@math.ubc.ca 
continuation of [20], here we shall carry out an error analysis of a fully discrete interior penalty (IP) discontinuous Galerkin (DG) finite element method (FEM) for the wave equation. For simplicity, we consider the following initial-boundary value model problem: find $u(x, t)$ such that

$$
\begin{array}{rlrl}
u_{t t}-\nabla \cdot\left(c^{2} \nabla u\right) & =f & & \text { in } \Omega \times J, \\
u=0 & & \text { on } \partial \Omega \times J, \\
\left.u\right|_{t=0}=u_{0} & & \text { in } \Omega, \\
\left.u_{t}\right|_{t=0}=v_{0} & & \text { in } \Omega .
\end{array}
$$

Here, $J=(0, T)$ is a finite time interval and $\Omega \subset \mathbb{R}^{d}, d=2,3$, is a bounded Lipschitz polygon $(d=2)$ or Lipschitz polyhedron $(d=3)$. The coefficient $c(x)$ is the wave speed, $f(x, t)$ is a given source term, and $u_{0}(x)$ and $v_{0}(x)$ are prescribed initial data.

Throughout this paper, we assume $\Omega$ to be convex, $f \in C\left(\bar{J} ; L^{2}(\Omega)\right), u_{0} \in H_{0}^{1}(\Omega), v_{0} \in$ $L^{2}(\Omega)$, and the function $c(x)$ to be a smooth function bounded from above and below:

$$
0<c_{\min } \leq c(x) \leq c_{\max }<\infty, \quad x \in \bar{\Omega} .
$$

A-priori error estimates for continuous Galerkin approximations of the wave equation (1)-(4) were first derived by Dupont [15] and later improved by Baker [3], both for continuous and discrete time schemes. Gekeler [18] analyzed general multi-step methods for the time discretization of second-order hyperbolic equations, when a Galerkin procedure is used in space. High-order accurate two-step approximations for second-order hyperbolic equations were developed in [4]. These schemes are based on rational approximations of the cosine function and require the solution of a number of linear systems in each time step. In [29] high-order Taylor-Galerkin schemes were presented for second-order hyperbolic problems and combined with an $h p$-adaptive strategy.

Mixed finite element methods were proposed for the wave equation in [12, 19]. Here convergence and stability typically require compatibility of the approximating spaces via the inf-sup condition. In [13] convergence results for explicit and implicit second-order accurate discrete time stepping are established. A new class of mixed finite elements on regular meshes was proposed in [5] and combined with the ficticious domain method and masslumping for efficiency. Continuous space-time finite elements were presented in [17]. They are based on tensor-product spaces for the full discretization and reduce to Gauss-Legendre implicit Runge-Kutta methods in the homogeneous case.

Standard continuous (conforming) Galerkin methods impose significant restrictions on the underlying mesh and discretization; in particular, they do not easily accommodate hanging nodes for local mesh refinement. In addition, if explicit time stepping is subsequently employed, the mass matrix arising from the spatial discretization by standard continuous finite elements must be inverted at each time step: a major drawback in terms of efficiency. For low-order Lagrange $\left(\mathcal{P}_{1}\right)$ elements, so-called mass lumping overcomes this problem [6, 25], but for higher-order elements this procedure can lead to unstable schemes unless particular finite elements and quadrature rules are used [11].

In contrast, discontinuous Galerkin (DG) methods easily handle elements of various types and shapes, irregular nonmatching grids, and even locally varying polynomial order. In DG methods, continuity is weakly enforced across mesh interfaces by adding suitable bilinear forms, so-called numerical fluxes, to standard variational formulations. Because individual elements decouple, DG-FEMs are also inherently parallel; see [7-10] for further details 
and recent reviews. Moreover, the mass matrix arising from the spatial DG discretization is block-diagonal, with block size equal to the number of degrees of freedom per element; it can therefore be inverted at very low computational cost. In fact, for a judicious choice of (locally orthogonal) shape functions, the mass matrix is diagonal. When combined with explicit time integration, the resulting time marching scheme will be fully explicit.

When applied to second-order hyperbolic problems, most DG methods first require the problem to be reformulated as a first-order hyperbolic system, for which various DG methods are available $[9,16,23,24,26]$. A first DG method for the acoustic wave equation in its original second-order formulation was recently proposed by Rivière and Wheeler [28]; it is based on a nonsymmetric interior penalty formulation and requires additional stabilization terms for optimal convergence in the $L^{2}$-norm [27].

In [20] a symmetric interior penalty discontinuous Galerkin method was presented for the time-dependent wave equation. In particular, optimal a-priori error bounds in the energy norm and the $L^{2}$-norm were derived for the semi-discrete formulation. Besides the wellknown advantages of DG methods mentioned above, a symmetric discretization of the wave equation in its second-order form offers an additional advantage, which also pertains to the classical continuous Galerkin formulation: since the stiffness matrix is symmetric positive definite, the semi-discrete formulation conserves (a discrete version of) the energy for all time. The dispersive properties of the symmetric interior penalty DG method were recently analyzed by Ainsworth, Monk, and Muniz [1].

Here we extend the error analysis to the fully discrete numerical scheme, when the popular explicit second-order "leap-frog" scheme is used for the time discretization. The wave equation is analyzed in its original second-order form, and so is the two-step leap-frog scheme. As a consequence, the CFL (Courant-Friedrichs-Lewy) stability condition naturally arises in the convergence proof. Some of the techniques used in the proof can be found in previous works $[3,13]$, but to the best of our knowledge, this proof has not appeared elsewhere in its present form, not even for the standard conforming case.

The rest of the paper is organized as follows. In Sect. 2, we present the discontinuous Galerkin discretization of the wave equation. In Sect. 3, we state an a-priori error estimate which is optimal in both space and time. The proof of this estimate is carried out in detail in Sect. 4. Finally, we end the paper with some concluding remarks in Sect. 5.

\section{Discontinuous Galerkin Discretization}

In this section, we present the fully discrete discontinuous Galerkin method for the wave equation. The discretization in space is based on the interior penalty method presented in [20] while the time discretization is based on a standard centered second-order finite difference ("leap-frog") approximation.

\subsection{Space Discretization}

To discretize the wave equation (1)-(4) in space, we consider regular and quasi-uniform meshes $\mathcal{T}_{h}=\{K\}$ of mesh size $h$ that partition the domain $\Omega$ into triangles $(d=2)$ or tetrahedra $(d=3)$. We denote by $\mathcal{F}_{h}^{I}$ the set of all interior edges or faces of $\mathcal{T}_{h}$, by $\mathcal{F}_{h}^{B}$ the set of all boundary edges or faces, and define $\mathcal{F}_{h}=\mathcal{F}_{h}^{I} \cup \mathcal{F}_{h}^{B}$. The diameter of element $K$ is denoted by $h_{K}$.

We will use standard notation for averages and jumps. To define it, let $\mathbf{u}$ be a piecewise smooth vector-valued function, and let $F \in \mathcal{F}_{h}^{\mathcal{I}}$ be an interior edge or face shared by two 
neighboring elements $K^{+}$and $K^{-}$. If we denote by $\mathbf{u}^{ \pm}$the trace of $\mathbf{u}$ taken from within $K^{ \pm}$, the average of $\mathbf{u}$ over $F$ is defined by

$$
\{\mathbf{u}\}=\frac{1}{2}\left(\mathbf{u}^{+}+\mathbf{u}^{-}\right)
$$

Similarly, for a scalar function $u$, the jump over $F$ is given by

$$
\llbracket u \rrbracket=u^{+} \mathbf{n}_{K^{+}}+u^{-} \mathbf{n}_{K^{-}},
$$

where $\mathbf{n}_{K^{ \pm}}$denotes the unit outward normal vector on the boundary $\partial K^{ \pm}$. For a boundary edge or face $F \in \mathcal{F}_{h}^{\mathcal{B}}$, we set $\{\mathbf{u}\}=\mathbf{u}$ and $\llbracket u \rrbracket=u \mathbf{n}$, where we denote by $\mathbf{n}$ the unit outward normal vector on $\partial \Omega$.

For a given mesh $\mathcal{T}_{h}$ and an approximation order $p \geq 1$, we define the discontinuous Galerkin finite element space

$$
V^{h}=\left\{u \in L^{2}(\Omega):\left.u\right|_{K} \in \mathcal{P}_{p}(K), K \in \mathcal{T}_{h}\right\},
$$

with $\mathcal{P}_{p}(K)$ denoting the polynomials of (total) degree less or equal than $p$.

We now consider the following discontinuous Galerkin method for the spatial discretization of (1)-(4): find $u^{h}: \bar{J} \times V^{h} \rightarrow \mathbb{R}$ such that

$$
\begin{aligned}
\left(u_{t t}^{h}, v\right)+a_{h}\left(u^{h}, v\right) & =(f, v), \quad v \in V^{h}, t \in J, \\
\left.u^{h}\right|_{t=0} & =P_{h} u_{0}, \\
\left.u_{t}^{h}\right|_{t=0} & =P_{h} v_{0} .
\end{aligned}
$$

Here, $P_{h}$ denotes the $L^{2}$-projection onto $V^{h}$. The discrete bilinear form $a_{h}$ is the standard symmetric interior penalty form for the Laplacian given by

$$
\begin{aligned}
a_{h}(u, v)= & \sum_{K \in \mathcal{T}_{h}} \int_{K} c^{2} \nabla u \cdot \nabla v d x-\sum_{F \in \mathcal{F}_{h}} \int_{F} \llbracket u \rrbracket \cdot \llbracket c^{2} \nabla v \rrbracket d s \\
& \left.-\sum_{F \in \mathcal{F}_{h}} \int_{F} \llbracket v \rrbracket \cdot \llbracket c^{2} \nabla u\right\} d s+\sum_{F \in \mathcal{F}_{h}} \gamma h_{F}^{-1} \int_{F} c^{2} \llbracket u \rrbracket \cdot \llbracket v \rrbracket d s,
\end{aligned}
$$

with $h_{F}$ denoting the diameter of the edge or the face $F$. The parameter $\gamma>0$ is the interior penalty stabilization parameter that has to be chosen sufficiently large, independently of the mesh size; see Lemma 3.2 below. We point out that the form $a_{h}$ is symmetric:

$$
a_{h}(u, v)=a_{h}(v, u), \quad u, v \in V^{h} .
$$

Remark 2.1 Our analysis immediately extends to other spatial DG discretizations as long as the following four key assumptions on the bilinear form $a_{h}$ hold: symmetry, continuity, coercivity and adjoint-consistency, cf. [2]. For instance, the popular local discontinuous Galerkin (LDG) discretization indeed satisfies all these assumptions. However, the constants appearing in the CFL condition (17) will slightly vary for different DG methods. 


\subsection{Time Discretization}

We now use the leap-frog scheme to discretize in time the system of ordinary differential equations (7)-(9). To that end we introduce a time step $\Delta t=T / N$ and define the discrete times $t_{n}=n \Delta t$ for $n=0, \ldots, N$. For a (sufficiently smooth) function $v(x, t)$, we set

$$
\partial_{t}^{i} v^{n}=\partial_{t}^{i} v\left(\cdot, t_{n}\right), \quad i \geq 0
$$

Let $u$ now be the solution to the wave equation (1)-(4). We wish to find DG approximations $\left\{U^{n}\right\}$ such that $U^{n} \approx u^{n}$ at the discrete times $t_{n}$. To do so, we introduce the finite difference operator

$$
\delta^{2} U^{n}=\frac{U^{n+1}-2 U^{n}+U^{n-1}}{\Delta t^{2}}, \quad n=1, \ldots, N-1 .
$$

The fully discrete numerical solution to the wave equation (1)-(4) is then defined by finding $\left\{U^{n}\right\}_{n=0}^{N}$ such that

$$
\left(\delta^{2} U^{n}, v\right)+a_{h}\left(U^{n}, v\right)=\left(f^{n}, v\right), \quad v \in V^{h}, n=1, \ldots, N-1 .
$$

The initial conditions $U^{0} \in V^{h}$ and $U^{1} \in V^{h}$ are given by

$$
\begin{aligned}
& U^{0}=P_{h} u_{0}, \\
& U^{1}=U^{0}+\Delta t P_{h} v_{0}+\frac{\Delta t^{2}}{2} \widetilde{U}^{0},
\end{aligned}
$$

with $\widetilde{U}^{0} \in V^{h}$ defined by

$$
\left(\widetilde{U}^{0}, v\right)=\left(f^{0}, v\right)-a_{h}\left(u_{0}, v\right), \quad v \in V^{h} .
$$

In (12), every time step involves the inversion of the DG mass matrix. Since it is symmetric positive definite, the new approximations $U^{n+1}$ are well-defined for $n \geq 1$. Therefore the fully discrete DG approximations $\left\{U^{n}\right\}_{n=0}^{N}$ are uniquely defined by (12)-(15), which completes the definition of the fully discrete DG method for the wave equation.

\section{An Optimal A-priori Error Estimate}

In this section, we state our main result: an optimal a-priori error estimate for the fully discrete DG method introduced above.

\subsection{Properties of the Bilinear Form $a_{h}$}

We first review the key stability properties of the bilinear form $a_{h}$. To that end, we introduce the broken norm

$$
\|u\|_{\star}^{2}=\sum_{K \in \mathcal{T}_{h}}\|\nabla u\|_{0, K}^{2}+\sum_{K \in \mathcal{T}_{h}} h_{K}^{2}\left\|D^{2} u\right\|_{0, K}^{2}+\sum_{F \in \mathcal{F}_{h}} h_{F}^{-1}\|\llbracket u \rrbracket\|_{0, F}^{2},
$$

with $D^{2} u$ denoting the matrix of the second derivatives of $u$. 
Remark 3.1 Let us point out that the norm $\|u\|_{\star}$ is the natural one for obtaining continuity of the bilinear form $a_{h}$ on $H^{2}(\Omega)+V^{h}$, while the weaker DG norm

$$
u \mapsto \sum_{K \in \mathcal{T}_{h}}\|\nabla u\|_{0, K}^{2}+\sum_{F \in \mathcal{F}_{h}} h_{F}^{-1}\|\llbracket u \rrbracket\|_{0, F}^{2}
$$

is enough for obtaining coercivity. Notice that, for discrete functions in $V^{h}$, these two norms are equivalent. For more details, we refer the reader to the discussion in Sect. 4 of [2].

The following results hold; cf. [2, Sects. 4.1 and 4.2] and [20].

Lemma 3.2 There exists a threshold value $\gamma_{0}>0$, independent of the mesh size, such that for $\gamma \geq \gamma_{0}$ there holds

$$
a_{h}(u, u) \geq C_{C}\|u\|_{\star}^{2}, \quad u \in V^{h},
$$

with a coercivity constant $C_{C}>0$ that is independent of the mesh size. Moreover, we have

$$
\left|a_{h}(u, v)\right| \leq C_{A} c_{\max }^{2} \max \{1, \gamma\}\|u\|_{\star}\|v\|_{\star}, \quad u, v \in H^{2}(\Omega)+V^{h},
$$

with a continuity constant $C_{A}>0$ that is independent of the mesh size, $c^{2}$ and $\gamma$.

Finally, the following spectral estimate will play a crucial role in our analysis.

Lemma 3.3 For quasi-uniform meshes $\mathcal{T}_{h}$, there holds

$$
a_{h}(u, u) \leq C_{S} c_{\max }^{2} \max \{1, \gamma\} h^{-2}\|u\|_{0}^{2}, \quad u \in V^{h},
$$

with a stability constant $C_{S}>0$ that is independent of the mesh size, $c^{2}$ and $\gamma$.

Proof Let $u \in V^{h}$. From the continuity of $a_{h}$ in Lemma 3.2, we have

$$
a_{h}(u, u) \leq C_{A} c_{\max }^{2} \max \{1, \gamma\}\|u\|_{\star}^{2} .
$$

For an element $K$ and $u \in \mathcal{P}_{p}(K)$, we recall the inverse estimates

$$
\|\nabla u\|_{0, K} \leq C h_{K}^{-1}\|u\|_{0, K}, \quad\left\|D^{2} u\right\|_{0, K} \leq C h_{K}^{-2}\|u\|_{0, K},
$$

as well as the trace estimate

$$
\|u\|_{0, \partial K} \leq C h_{K}^{-\frac{1}{2}}\|u\|_{0, K},
$$

with a constant $C>0$ that only depends on the shape-regularity constants of the meshes and the polynomial degree $p$. These estimates and the quasi-uniformity of the meshes immediately show that

$$
\|u\|_{\star}^{2} \leq C_{I} h^{-2}\|u\|_{0}^{2},
$$

with a constant $C_{I}>0$ that is independent of the mesh size. Combining the above estimates gives

$$
a_{h}(u, u) \leq C_{I} C_{A} c_{\max }^{2} \max \{1, \gamma\} h^{-2}\|u\|_{0}^{2},
$$

which is the desired bound with stability constant $C_{S}=C_{I} C_{A}$. 


\subsection{An Optimal A-priori Error Estimate}

We are now ready to state our error estimate for the fully discrete DG method. We suppose that the mesh size $h$ and the time step $\Delta t$ satisfy the CFL condition

$$
\Delta t<\frac{2 h}{c_{\max } \sqrt{C_{S} \max \{1, \gamma\}}},
$$

where $\gamma \geq \gamma_{0}$ is the threshold parameter from Lemma 3.2 and $C_{S}$ is the constant of Lemma 3.3.

The following result holds.

Theorem 3.4 Let the solution u of the wave equation (1)-(4) satisfy the regularity properties

$$
u \in C^{2}\left(\bar{J} ; H^{p+1}(\Omega)\right), \quad \partial_{t}^{3} u \in C\left(\bar{J} ; L^{2}(\Omega)\right), \quad \partial_{t}^{4} u \in L^{1}\left(J ; L^{2}(\Omega)\right) .
$$

Furthermore, let the discrete DG approximations $\left\{U^{n}\right\}_{n=0}^{N}$ be defined by (12)-(15) and assume that the CFL condition (17) is satisfied. Then there holds the error estimate

$$
\max _{n=0}^{N}\left\|u^{n}-U^{n}\right\|_{0} \leq C\left(h^{p+1}+\Delta t^{2}\right)
$$

with a constant $C>0$ that is independent of the mesh size and the time step.

Remark 3.5 A close inspection of the proof shows that the constant $C$ in Theorem 3.4 grows linearly with $T$.

\section{Convergence Proof}

In this section, we present the proof of Theorem 3.4.

\subsection{Galerkin Projection}

Let $u \in H^{2}(\Omega)$. The Galerkin projection $\pi_{h} u \in V^{h}$ of $u$ is defined by requiring that

$$
a_{h}\left(\pi_{h} u, v\right)=a_{h}(u, v) \quad \forall v \in V^{h} .
$$

The following approximation properties hold.

Lemma 4.1 If additionally $u \in H^{p+1}(\Omega)$ for $p \geq 1$, then

$$
\begin{aligned}
& \left\|u-\pi_{h} u\right\|_{\star} \leq C h^{p}\|u\|_{p+1}, \\
& \left\|u-\pi_{h} u\right\|_{0} \leq C h^{p+1}\|u\|_{p+1},
\end{aligned}
$$

with a constant $C>0$ that is independent of the mesh size.

Proof The stability results in Lemma 3.2 readily imply that

$$
\left\|u-\pi_{h} u\right\|_{\star} \leq C \inf _{v \in V^{h}}\|u-v\|_{\star} .
$$


Standard approximation properties then yield the first estimate.

To prove the second estimate, we use a duality argument and consider the auxiliary problem

$$
-\nabla \cdot\left(c^{2} \nabla z\right)=u-\pi_{h} u \quad \text { in } \Omega, \quad z=0 \quad \text { on } \partial \Omega .
$$

Since we assumed the domain $\Omega$ to be convex and $c^{2}$ to be smooth, elliptic regularity gives $z \in H^{2}(\Omega) \cap H_{0}^{1}(\Omega)$ and $\|z\|_{2} \leq C\left\|u-\pi_{h} u\right\|_{0}$. Moreover, the interior penalty form $a_{h}$ is adjoint consistent; see [2, Sect. 3.3]. This implies that

$$
\left\|u-\pi_{h} u\right\|_{0}^{2}=a_{h}\left(u-\pi_{h} u, z\right)=a_{h}\left(u-\pi_{h} u, z-z_{h}\right) \leq C\left\|u-\pi_{h} u\right\|_{\star}\left\|z-z_{h}\right\|_{\star}
$$

for any $z_{h} \in V^{h}$. Choosing $z_{h}$ to be the $L^{2}$-projection yields

$$
\left\|z-z_{h}\right\|_{\star} \leq C h\|z\|_{2} \leq C h\left\|u-\pi_{h} u\right\|_{0} .
$$

We conclude that

$$
\left\|u-\pi_{h} u\right\|_{0} \leq C h\left\|u-\pi_{h} u\right\|_{\star},
$$

which yields the desired $L^{2}$-bound for the Galerkin projection.

Let the solution $u$ of the wave equation satisfy the regularity assumptions in Theorem 3.4. We define $\pi_{h} u(\cdot, t) \in V^{h}$ by requesting that

$$
a_{h}\left(\pi_{h} u(\cdot, t), v\right)=a_{h}(u(\cdot, t), v) \quad \forall v \in V^{h} .
$$

Since

$$
\partial_{t}^{i}\left(\pi_{h} u\right)=\pi_{h}\left(\partial_{t}^{i} u\right), \quad i=0, \ldots, 2,
$$

the following result is an immediate consequence of Lemma 4.1.

Lemma 4.2 Let $u$ satisfy the regularity properties in Theorem 3.4 and let $\pi_{h} u$ be defined by (19). Then we have

$$
\left\|\partial_{t}^{i}\left(u-\pi_{h} u\right)\right\|_{0} \leq C h^{p+1}\left\|\partial_{t}^{i} u\right\|_{p+1}, \quad i=0, \ldots, 2, t \in \bar{J},
$$

with a constant $C>0$ that is independent of the mesh size.

\subsection{Error Bound}

We decompose the error $e^{n}=u^{n}-U^{n}$ at time $t_{n}$ into

$$
e^{n}=u^{n}-w^{n}+w^{n}-U^{n}=\eta^{n}+\phi^{n}, \quad n=0, \ldots, N,
$$

where $w^{n}=\pi_{h} u^{n} \in V^{h}$ is the Galerkin projection of $u^{n}$. In order to derive an error bound, we set

$$
r^{n}= \begin{cases}\delta^{2} w^{n}-u_{t t}^{n}, & n=1, \ldots, N-1, \\ \Delta t^{-2}\left(\phi^{1}-\phi^{0}\right), & n=0 .\end{cases}
$$

We then define

$$
R^{n}=\Delta t \sum_{m=0}^{n} r^{m}
$$


The following error bound holds.

Proposition 4.3 Assume that the CFL condition (17) holds. Then we have

$$
\max _{n=0}^{N}\left\|e^{n}\right\|_{0} \leq C\left(\left\|e^{0}\right\|_{0}+\max _{n=0}^{N}\left\|\eta^{n}\right\|_{0}+\Delta t \sum_{n=0}^{N-1}\left\|R^{n}\right\|_{0}\right),
$$

with a constant $C>0$ that is independent of $h, \Delta t$ and $T$.

Proof By the triangle inequality, we have that

$$
\max _{n=0}^{N}\left\|e^{n}\right\|_{0} \leq \max _{n=0}^{N}\left\|\phi^{n}\right\|_{0}+\max _{n=0}^{N}\left\|\eta^{n}\right\|_{0},
$$

and need to further bound $\max _{n=0}^{N}\left\|\phi^{n}\right\|_{0}$.

To that end, we first notice that, under the regularity assumptions in Theorem 3.4, the exact solution $u$ to the wave equation satisfies

$$
\left(u_{t t}^{n}, v\right)+a_{h}\left(u^{n}, v\right)=\left(f^{n}, v\right), \quad v \in V^{h}, n=1, \ldots, N .
$$

This follows readily from the consistency of the bilinear form $a_{h}$; cf. [2, Sect. 3.3].

Next, we subtract (12) from (25) and conclude that

$$
\left(u_{t t}^{n}-\delta^{2} w^{n}+\delta^{2} w^{n}-\delta^{2} U^{n}, v\right)+a_{h}\left(u^{n}-w^{n}+w^{n}-U^{n}, v\right)=0
$$

for all $v \in V^{h}$ and $n=1, \ldots, N-1$. Since $a_{h}\left(u^{n}-w^{n}, v\right)=0$ by the definition of the Galerkin projection, we obtain that

$$
\left(\delta^{2} \phi^{n}, v\right)+a_{h}\left(\phi^{n}, v\right)=\left(r^{n}, v\right)
$$

for all $v \in V^{h}$ and $n=1, \ldots, N-1$. We now add up (26) from $n=1$ to $n=m$, for $1 \leq m \leq$ $N-1$. Taking into account cancelation and multiplying with $\Delta t$, we readily see that

$$
\left(\frac{\phi^{m+1}-\phi^{m}}{\Delta t}, v\right)-\left(\frac{\phi^{1}-\phi^{0}}{\Delta t}, v\right)+\Delta t \sum_{n=1}^{m} a_{h}\left(\phi^{n}, v\right)=\Delta t \sum_{n=1}^{m}\left(r^{n}, v\right) \text {. }
$$

Upon defining

$$
\Phi^{m}=\Delta t \sum_{n=1}^{m} \phi^{n}, \quad \Phi^{0}=0
$$

we have

$$
\left(\frac{\phi^{m+1}-\phi^{m}}{\Delta t}, v\right)+a_{h}\left(\Phi^{m}, v\right)=\left(R^{m}, v\right), \quad v \in V^{h}, 0 \leq m \leq N-1
$$

Next, we choose $v=\phi^{m+1}+\phi^{m} \in V^{h}$ above and multiply the resulting expression by $\Delta t$. This yields

$$
\left\|\phi^{m+1}\right\|_{0}^{2}-\left\|\phi^{m}\right\|_{0}^{2}+\Delta t a_{h}\left(\Phi^{m}, \phi^{m}+\phi^{m+1}\right)=\Delta t\left(R^{m}, \phi^{m}+\phi^{m+1}\right),
$$


for $0 \leq m \leq N-1$. Summation from $m=0$ to $m=n-1$, for $1 \leq n \leq N$, shows that

$$
\left\|\phi^{n}\right\|_{0}^{2}-\left\|\phi^{0}\right\|_{0}^{2}+\Delta t \sum_{m=0}^{n-1} a_{h}\left(\Phi^{m}, \phi^{m}+\phi^{m+1}\right)=\Delta t \sum_{m=0}^{n-1}\left(R^{m}, \phi^{m}+\phi^{m+1}\right) .
$$

Since $a_{h}$ is symmetric, $\Phi^{0}=0$, and

$$
\Phi^{m+1}-\Phi^{m-1}=\Delta t\left(\phi^{m}+\phi^{m+1}\right), \quad m=1, \ldots, N-1,
$$

we conclude that

$$
\begin{aligned}
\Delta t \sum_{m=0}^{n-1} a_{h}\left(\Phi^{m}, \phi^{m}+\phi^{m+1}\right) & =\sum_{m=1}^{n-1} a_{h}\left(\Phi^{m}, \Phi^{m+1}-\Phi^{m-1}\right) \\
& =\sum_{m=1}^{n-1} a_{h}\left(\Phi^{m}, \Phi^{m+1}\right)-\sum_{m=0}^{n-2} a_{h}\left(\Phi^{m+1}, \Phi^{m}\right) \\
& =a_{h}\left(\Phi^{n-1}, \Phi^{n}\right)
\end{aligned}
$$

By the symmetry and the coercivity properties of $a_{h}$, and since $\Phi^{n}-\Phi^{n-1}=\Delta t \phi^{n}$ for $n=1, \ldots, N$, we further find that

$$
\begin{aligned}
a_{h}\left(\Phi^{n-1}, \Phi^{n}\right)= & a_{h}\left(\frac{\Phi^{n-1}+\Phi^{n}}{2}, \frac{\Phi^{n-1}+\Phi^{n}}{2}\right) \\
& -a_{h}\left(\frac{\Phi^{n}-\Phi^{n-1}}{2}, \frac{\Phi^{n}-\Phi^{n-1}}{2}\right) \\
\geq & -\frac{\Delta t^{2}}{4} a_{h}\left(\phi^{n}, \phi^{n}\right) .
\end{aligned}
$$

Hence, we conclude that

$$
\left\|\phi^{n}\right\|_{0}^{2}-\frac{\Delta t^{2}}{4} a_{h}\left(\phi^{n}, \phi^{n}\right) \leq\left\|\phi^{0}\right\|_{0}^{2}+\Delta t \sum_{m=0}^{n-1}\left(R^{m}, \phi^{m}+\phi^{m+1}\right),
$$

for $1 \leq n \leq N$. The estimate in Lemma 3.3 now yields

$$
a_{h}\left(\phi^{n}, \phi^{n}\right) \leq C_{S} c_{\max }^{2} \max \{1, \gamma\} h^{-2}\left\|\phi^{n}\right\|_{0}^{2} .
$$

Therefore, if the CFL condition (17) holds, we have

$$
C_{\star}\left\|\phi^{n}\right\|_{0}^{2} \leq\left\|\phi^{0}\right\|_{0}^{2}+\Delta t \sum_{m=0}^{n-1}\left(R^{m}, \phi^{m}+\phi^{m+1}\right), \quad 1 \leq n \leq N,
$$

with

$$
C_{\star}=1-\frac{\Delta t^{2}}{4} C_{S} c_{\max }^{2} \max \{1, \gamma\} h^{-2}>0 .
$$


By using the Cauchy-Schwarz inequality and the geometric-arithmetic inequality in (29), we find that

$$
\begin{aligned}
C_{\star}\left\|\phi^{n}\right\|_{0}^{2} & \leq\left\|\phi^{0}\right\|_{0}^{2}+2\left(\max _{n=0}^{N}\left\|\phi^{n}\right\|_{0}\right)\left(\Delta t \sum_{n=0}^{N-1}\left\|R^{n}\right\|_{0}\right) \\
& \leq\left\|\phi^{0}\right\|_{0}^{2}+\frac{C_{\star}}{2} \max _{n=0}^{N}\left\|\phi^{n}\right\|_{0}^{2}+\frac{2}{C_{\star}}\left(\Delta t \sum_{n=0}^{N-1}\left\|R^{n}\right\|_{0}\right)^{2},
\end{aligned}
$$

for $0 \leq n \leq N$. Since the right-hand side is independent of $n$, we readily obtain that

$$
\begin{aligned}
C_{\star} \max _{n=0}^{N}\left\|\phi^{n}\right\|_{0}^{2} & \leq 2\left\|\phi^{0}\right\|_{0}^{2}+\frac{4}{C_{\star}}\left(\Delta t \sum_{n=0}^{N-1}\left\|R^{n}\right\|_{0}\right)^{2} \\
& \leq\left(\sqrt{2}\left\|\phi^{0}\right\|_{0}+\frac{2 \Delta t}{\sqrt{C_{\star}}} \sum_{n=0}^{N-1}\left\|R^{n}\right\|_{0}\right)^{2} .
\end{aligned}
$$

Taking square roots on both sides and dividing by $\sqrt{C_{\star}}$ shows that

$$
\max _{n=0}^{N}\left\|\phi^{n}\right\|_{0} \leq \sqrt{\frac{2}{C_{\star}}}\left\|\phi^{0}\right\|_{0}+\frac{2 \Delta t}{C_{\star}} \sum_{n=0}^{N-1}\left\|R^{n}\right\|_{0} .
$$

The desired estimate now follows immediately from (24), (31) and the fact that

$$
\left\|\phi^{0}\right\|_{0} \leq\left\|e^{0}\right\|_{0}+\left\|\eta^{0}\right\|_{0}
$$

This completes the proof.

We now bound the terms $\left\|R^{n}\right\|_{0}$ on the right-hand side of Proposition 4.3. To do so, we first estimate the $L^{2}$-norms of the functions $r^{n}$. We distinguish the cases $n=0$ and $n \geq 1$.

\section{Lemma 4.4 There holds}

$$
\left\|r^{0}\right\|_{0} \leq C\left(\Delta t^{-1} h^{p+1}\left\|u_{t}\right\|_{C\left(\bar{J} ; H^{p+1}(\Omega)\right)}+\Delta t\left\|u_{t t t}\right\|_{C\left(\bar{J} ; L^{2}(\Omega)\right)}\right),
$$

with a constant $C>0$ that is independent of $h, \Delta t$ and $T$.

Proof We recall from (22) that $r^{0}=\Delta t^{-2}\left(\phi^{1}-\phi^{0}\right)$. To estimate $\left\|\phi^{1}-\phi^{0}\right\|_{0}$, take $v \in V^{h}$ arbitrary. We then note that

$$
\begin{aligned}
\left(\phi^{1}-\phi^{0}, v\right) & =\left(w^{1}-U^{1}, v\right)-\left(w^{0}-U^{0}, v\right) \\
& =\left(w^{1}-u^{1}, v\right)+\left(u^{1}-U^{1}, v\right)-\left(w^{0}-u^{0}, v\right)-\left(u^{0}-U^{0}, v\right) \\
& =\left(\left(\pi_{h}-I\right)\left(u^{1}-u^{0}\right), v\right)+\left(u^{1}-U^{1}, v\right)
\end{aligned}
$$

where we have used that $\left(u^{0}-U^{0}, v\right)=\left(u_{0}-P_{h} u_{0}, v\right)=0$. 
We first estimate the term $\left(\left(\pi_{h}-I\right)\left(u^{1}-u^{0}\right), v\right)$ in (32). To do so, we use (20) and Lemma 4.2. We obtain

$$
\begin{aligned}
\left|\left(\left(\pi_{h}-I\right)\left(u^{1}-u^{0}\right), v\right)\right| & \leq \int_{0}^{t_{1}}\left|\left(\partial_{t}\left(\pi_{h}-I\right) u(., s), v\right)\right| d s \\
& \leq \int_{0}^{t_{1}}\left|\left(\left(\pi_{h}-I\right) u_{t}(., s), v\right)\right| d s \\
& \leq C \Delta t h^{p+1}\left\|u_{t}\right\|_{C\left(\bar{J} ; H^{p+1}(\Omega)\right)}\|v\|_{0} .
\end{aligned}
$$

Next, we estimate the term $\left(u^{1}-U^{1}, v\right)$ in (32). From Taylor's formula and since $u^{0}=u_{0}$, $u_{t}^{0}=v_{0}$, we have

$$
u^{1}=u_{0}+\Delta t v_{0}+\frac{\Delta t^{2}}{2} u_{t t}^{0}+\frac{1}{2} \int_{0}^{t_{1}}(\Delta t-s)^{2} u_{t t t}(\cdot, s) d s .
$$

From the definition of $U^{1}$ in (14) and the fact that

$$
\left(u_{0}-P_{h} u_{0}, v\right)=0, \quad\left(v_{0}-P_{h} v_{0}, v\right)=0,
$$

we deduce that

$$
\left(u^{1}-U^{1}, v\right)=\frac{\Delta t^{2}}{2}\left(u_{t t}^{0}-\widetilde{U}^{0}, v\right)+\frac{1}{2} \int_{0}^{t_{1}}(\Delta t-s)^{2}\left(u_{t t t}(\cdot, s), v\right) d s .
$$

The definition of $\widetilde{U}^{0}$ in (15) and the consistency of the DG method in (25) yield

$$
\left(u_{t t}^{0}-\widetilde{U}^{0}, v\right)=\left(f^{0}, v\right)-a_{h}\left(u_{0}, v\right)-\left(f^{0}, v\right)+a_{h}\left(u_{0}, v\right)=0 .
$$

Therefore,

$$
\begin{aligned}
\left|\left(u^{1}-U^{1}, v\right)\right| & \leq \frac{1}{2} \int_{0}^{t_{1}}(\Delta t-s)^{2}\left|\left(u_{t t t}(\cdot, s), v\right)\right| d s \\
& \leq C \Delta t^{3}\left\|u_{t t t}\right\|_{C\left(\bar{J} ; L^{2}(\Omega)\right)}\|v\|_{0} .
\end{aligned}
$$

Since $\phi^{1}-\phi^{0} \in V^{h}$, referring to (32), (33) and (34) yields

$$
\left\|\phi^{1}-\phi^{0}\right\|_{0} \leq C\left(\Delta t h^{p+1}\left\|u_{t}\right\|_{C\left(\bar{J} ; H^{p+1}(\Omega)\right)}+\Delta t^{3}\left\|u_{t t t}\right\|_{C\left(\bar{J} ; L^{2}(\Omega)\right)}\right) .
$$

Dividing (35) by $\Delta t^{-2}$ yields the desired estimate for $\left\|r^{0}\right\|_{0}$.

Lemma 4.5 For $1 \leq n \leq N-1$, there holds

$$
\left\|r^{n}\right\|_{0} \leq C\left(\frac{h^{p+1}}{\Delta t} \int_{t_{n-1}}^{t_{n+1}}\left\|u_{t t}(\cdot, s)\right\|_{p+1} d s+\Delta t \int_{t_{n-1}}^{t_{n+1}}\left\|\partial_{t}^{4} u\right\|_{0} d s\right),
$$

with a constant $C>0$ that is independent of $h, \Delta t$ and $T$.

Proof By the triangle inequality, we have

$$
\left\|r^{n}\right\|_{0}=\left\|\delta^{2} w^{n}-u_{t t}^{n}\right\|_{0} \leq\left\|\delta^{2}\left(\pi_{h}-I\right) u^{n}\right\|_{0}+\left\|\delta^{2} u^{n}-u_{t t}^{n}\right\|_{0} .
$$


To bound the first term on the right-hand side of (36), we use the identity

$$
v\left(\cdot, t_{n+1}\right)-2 v\left(\cdot, t_{n}\right)+v\left(\cdot, t_{n-1}\right)=\Delta t \int_{t_{n-1}}^{t_{n+1}}\left(1-\frac{\left|s-t_{n}\right|}{\Delta t}\right) v_{t t}(\cdot, s) d s,
$$

which is proved by integration by parts. By using property (20) and Lemma 4.2 we obtain

$$
\begin{aligned}
\left\|\delta^{2}\left(\pi_{h}-I\right) u^{n}\right\|_{0} & \leq \frac{1}{\Delta t} \int_{t_{n-1}}^{t_{n+1}}\left(1-\frac{\left|s-t_{n}\right|}{\Delta t}\right)\left\|\partial_{t}^{2}\left(\pi_{h}-I\right) u\right\|_{0} d s \\
& \leq C \frac{h^{p+1}}{\Delta t} \int_{t_{n-1}}^{t_{n+1}}\left\|u_{t t}(\cdot, s)\right\|_{p+1} d s .
\end{aligned}
$$

To estimate the second term on the right-hand side of (36), we use the identity

$$
\delta^{2} u^{n}-u_{t t}^{n}=\frac{1}{6 \Delta t^{2}} \int_{t_{n-1}}^{t_{n+1}}\left(\Delta t-\left|s-t_{n}\right|\right)^{3} \partial_{t}^{4} u(\cdot, s) d s,
$$

which obtained from Taylor's formula with integral remainder.

Since $\left(\Delta t-\left|s-t_{n}\right|\right) \leq \Delta t$, we deduce that

$$
\left\|\delta^{2} u^{n}-u_{t t}^{n}\right\|_{0} \leq \frac{\Delta t}{6} \int_{t_{n-1}}^{t_{n+1}}\left\|\partial_{t}^{4} u\right\|_{0} d s .
$$

Referring to (36), (37) and (38) shows the desired bound for $\left\|r^{n}\right\|_{0}$.

We are now ready to prove the following result.

Proposition 4.6 For $0 \leq n \leq N-1$, there holds

$$
\begin{aligned}
\left\|R^{n}\right\|_{0} \leq & C \Delta t^{2}\left(\left\|u_{t t t}\right\|_{C\left(\bar{J} ; L^{2}(\Omega)\right)}+\left\|\partial_{t}^{4} u\right\|_{L^{1}\left(J ; L^{2}(\Omega)\right)}\right) \\
& +C h^{p+1}\left(\left\|u_{t}\right\|_{C\left(\bar{J} ; H^{p+1}(\Omega)\right)}+\left\|u_{t t}\right\|_{C\left(\bar{J} ; H^{p+1}(\Omega)\right)}\right),
\end{aligned}
$$

with a constant $C>0$ that is independent of $h, \Delta t$ and $T$.

Proof Using the bounds for $\left\|r^{n}\right\|_{0}$ in Lemma 4.4 and Lemma 4.5, we obtain

$$
\begin{aligned}
\left\|R^{n}\right\|_{0} \leq & \Delta t\left\|r^{0}\right\|_{0}+\Delta t \sum_{m=1}^{N-1}\left\|r^{m}\right\|_{0} \\
\leq & C \Delta t^{2}\left(\left\|u_{t t t}\right\|_{C\left(\bar{J} ; L^{2}(\Omega)\right)}+\left\|\partial_{t}^{4} u\right\|_{L^{1}\left(J ; L^{2}(\Omega)\right)}\right) \\
& +C h^{p+1}\left(\left\|u_{t}\right\|_{C\left(\bar{J} ; H^{p+1}(\Omega)\right)}+\left\|u_{t t}\right\|_{C\left(\bar{J} ; H^{p+1}(\Omega)\right)}\right)
\end{aligned}
$$

This completes the proof.

\subsection{Proof of Theorem 3.4}

We are now ready to prove the error estimate in Theorem 3.4. We start from Proposition 4.3. Noting that

$$
\Delta t \sum_{n=0}^{N-1}\left\|R^{n}\right\|_{0} \leq T \max _{n=0}^{N-1}\left\|R^{n}\right\|_{0}
$$


we obtain

$$
\max _{n=0}^{N}\left\|e^{n}\right\|_{0} \leq C\left(\left\|e^{0}\right\|_{0}+\max _{n=0}^{N}\left\|\eta^{n}\right\|_{0}+T \max _{n=0}^{N-1}\left\|R^{n}\right\|_{0}\right) .
$$

By Lemma 4.2,

$$
\max _{n=0}^{N}\left\|\eta^{n}\right\|_{0} \leq C h^{p+1}\|u\|_{C\left(\bar{J} ; H^{p+1}(\Omega)\right)} .
$$

Furthermore, the approximation properties of the $L^{2}$-projection show that

$$
\left\|e^{0}\right\|_{0} \leq C h^{p+1}\left\|u_{0}\right\|_{p+1} \leq C h^{p+1}\|u\|_{C\left(\bar{J} ; H^{p+1}(\Omega)\right)} .
$$

Finally, we apply Proposition 4.6 to bound $\max _{n=0}^{N-1}\left\|R^{n}\right\|_{0}$. This results in

$$
\begin{aligned}
\max _{n=1}^{N}\left\|e^{n}\right\|_{0} \leq & C h^{p+1}\|u\|_{C^{2}\left(\bar{J} ; H^{p+1}(\Omega)\right)} \\
& +C \Delta t^{2}\left(\left\|u_{t t t}\right\|_{C\left(\bar{J} ; L^{2}(\Omega)\right)}+\left\|\partial_{t}^{4} u\right\|_{L^{1}\left(J ; L^{2}(\Omega)\right)}\right),
\end{aligned}
$$

which completes the proof of Theorem 3.4. Clearly, the constant $C$ in Theorem 3.4 grows linearly with $T$.

\section{Conclusions}

We have proved optimal a-priori error estimates for a symmetric interior penalty discontinuous Galerkin method [20], when the popular second-order leap-frog scheme is used for time discretization. For sufficiently smooth solutions, the maximal error in the $L^{2}$-norm error across the entire time interval converges optimally as $O\left(h^{p+1}+\Delta t^{2}\right)$ where $p$ denotes the polynomial degree, $h$ the mesh size, and $\Delta t$ the time step. Our convergence results hold for any fully discrete DG method where the underlying DG bilinear form is symmetric, continuous, coercive, and adjoint consistent in the sense of [2].

Because the mass matrix is block-diagonal, with block size given by the number of degrees of freedom per element, the numerical method is truly explicit and inherently parallel. As the stiffness matrix induced by the DG bilinear form is symmetric, the numerical method conserves (a discrete version of) the energy for all time under the stability condition (17), which arises naturally in the convergence proof-see [14] for numerical results that demonstrate energy conservation. When mesh refinement is restricted to a small region, the use of implicit methods, or a very small time step in the entire computational domain, are very high a price to pay. To overcome that stability restriction, explicit local time stepping schemes were recently proposed in [14], which also conserve the energy.

The analysis presented in this article can be extended to non-convex domains. In this case, the norm $\|\cdot\|_{\star}$ has to be replaced by the weaker DG norm mentioned in Remark 3.1. As in [20], the continuity of a perturbed bilinear forms can then be ensured by the introduction of lifting operators. However, since the crucial estimate for the Galerkin projection in Lemma 4.2 is based on duality, it will now be suboptimal in the mesh size. As a consequence, the error estimate of the fully discrete method will be suboptimal in the mesh size as well. The analysis can further be extended to the interior penalty DG methods for Maxwell's wave equations proposed in $[21,22]$, provided that the solutions are sufficiently smooth in space 
and time. Finally, it can be extended to higher-order two-step time integration methods, such as the fourth-order modified equation approach in [30].

\section{References}

1. Ainsworth, M., Monk, P., Muniz, W.: Dispersive and dissipative properties of discontinuous Galerkin finite element methods for the second-order wave equation. J. Sci. Comput. 27, 5-40 (2006)

2. Arnold, D.N., Brezzi, F., Cockburn, B., Marini, L.D.: Unified analysis of discontinuous Galerkin methods for elliptic problems. SIAM J. Numer. Anal. 39, 1749-1779 (2002)

3. Baker, G.A.: Error estimates for finite element methods for second-order hyperbolic equations. SIAM J. Numer. Anal. 13, 564-576 (1976)

4. Baker, G.A., Dougalis, V.A., Serbin, S.M.: High order accurate two-step approximations for hyperbolic equations. RAIRO. Modél. Math. Anal. Numér. 13, 201-226 (1979)

5. Bécache, E., Joly, P., Tsogka, C.: An analysis of new mixed finite elements for the approximation of wave propagation problems. SIAM J. Numer. Anal. 37, 1053-1084 (2000)

6. Ciarlet, P.G.: The Finite Element Method for Elliptic Problems. North-Holland, Amsterdam (1978)

7. Cockburn, B.: Discontinuous Galerkin methods for convection-dominated problems. In: Barth, T., Deconink, H. (eds.) High-Order Methods for Computational Physics. Lect. Notes Comput. Sci. Engrg., vol. 9, pp. 69-224. Springer, Berlin (1999)

8. Cockburn, B., Karniadakis, G.E., Shu, C.-W.: The development of discontinuous Galerkin methods. In: Cockburn, B., Karniadakis, G.E., Shu, C.-W. (eds.) Discontinuous Galerkin Methods: Theory, Computation and Applications. Lect. Notes Comput. Sci. Engrg., vol. 11, pp. 3-50. Springer, Berlin (2000)

9. Cockburn, B., Shu, C.-W.: TVB Runge-Kutta local projection discontinuous Galerkin method for conservation laws II: General framework. Math. Comput. 52, 411-435 (1989)

10. Cockburn, B., Shu, C.-W.: Runge-Kutta discontinuous Galerkin methods for convection-dominated problems. J. Sci. Comput. 16, 173-261 (2001)

11. Cohen, G., Joly, P., Roberts, J.E., Tordjman, N.: Higher order triangular finite elements with mass lumping for the wave equation. SIAM J. Numer. Anal. 38, 2047-2078 (2001)

12. Cowsar, L.C., Dupont, T.F., Wheeler, M.F.: A-priori estimates for mixed finite element methods for the wave equations. Comput. Methods Appl. Mech. Eng. 82, 205-222 (1990)

13. Cowsar, L.C., Dupont, T.F., Wheeler, M.F.: A-priori estimates for mixed finite element approximations of second-order hyperbolic equations with absorbing boundary conditions. SIAM J. Numer. Anal. 33, 492-504 (1996)

14. Diaz, J., Grote, M.J.: Energy conserving explicit local time-stepping for second-order wave equations. SIAM J. Sci. Comput. (in press)

15. Dupont, T.: $L^{2}$-estimates for Galerkin methods for second-order hyperbolic equations. SIAM J. Numer. Anal. 10, 880-889 (1973)

16. Falk, R.S., Richter, G.R.: Explicit finite element methods for symmetric hyperbolic equations. SIAM J. Numer. Anal. 36(3), 935-952 (1999)

17. French, D.A., Peterson, T.E.: A continuous space-time finite element method for the wave equation. Math. Comput. 66, 491-506 (1996)

18. Gekeler, E.: Linear multi-step methods and Galerkin procedures for initial boundary value problems. SIAM J. Numer. Anal. 13, 536-548 (1976)

19. Geveci, T.: On the application of mixed finite methods to the wave equation. RAIRO Modél. Math. Anal. Numér. 22, 243-250 (1988)

20. Grote, M.J., Schneebeli, A., Schötzau, D.: Discontinuous Galerkin finite element method for the wave equation. SIAM J. Numer. Anal. 44, 2408-2431 (2006)

21. Grote, M.J., Schneebeli, A., Schötzau, D.: Interior penalty discontinuous Galerkin method for Maxwell's equations: Energy norm error estimates. J. Comput. Appl. Math. 204, 375-386 (2007)

22. Grote, M.J., Schneebeli, A., Schötzau, D.: Interior penalty discontinuous Galerkin method for Maxwell's equations: Optimal $L^{2}$-norm error estimates. IMA J. Numer. Anal. 28, 440-468 (2008)

23. Hesthaven, J.S., Warburton, T.: Nodal high-order methods on unstructured grids I: Time-domain solution of Maxwell's equations. J. Comput. Phys. 181, 186-221 (2002)

24. Houston, P., Jensen, M., Süli, E.: $h p$-discontinuous Galerkin finite element methods with least-squares stabilization. J. Sci. Comput. 17, 3-25 (2002)

25. Hughes, T.: The Finite Element Method: Linear Static and Dynamic Finite Element Analysis. Prentice Hall, New York (1987) 
26. Monk, P., Richter, G.R.: A discontinuous Galerkin method for linear symmetric hyperbolic systems in inhomogeneous media. J. Sci. Comput. 22-23, 443-477 (2005)

27. Rivière, B., Wheeler, M.F.: Discontinuous finite element methods for acoustic and elastic wave problems I: Semi-discrete error estimates. Technical Report 01-02, TICAM, UT Austin (2001)

28. Rivière, B., Wheeler, M.F.: Discontinuous finite element methods for acoustic and elastic wave problems. In: ICM2002-Beijing Satellite Conference on Scientific Computing. Contemporary Mathematics, vol. 329, pp. 271-282. Am. Math. Soc., Providence (2003)

29. Safjan, A., Oden, J.T.: High-order Taylor-Galerkin and adaptive $h-p$ methods for second-order hyperbolic systems: Applications to elastodynamics. Comput. Methods Appl. Mech. Eng. 103, 187-230 (1993)

30. Shubin, G.R., Bell, J.: A modified equation approach to constructing fourth-order methods for acoustic wave propagation. SIAM J. Sci. Comput. 8, 135-151 (1987) 